\title{
Opportunities in Integration of Family Planning in HIV/AIDS Services in Nepal
}

\author{
Mishra SR $^{1}$ \\ ${ }^{1}$ Journal Office
}

Health Prospect, Volume 11

\section{Introduction}

Integration of health services has been defined as offering a range of services that can meet several needs simultaneously, usually at the same time, in the same venue, and through the same provider. (1) Referrals to, or linkages with, other related services enable a client to receive a range of needed services, even if the services are not received simultaneously. This situation provides opportunities to reach a substantial proportion of the sexually active population that is seeking to prevent pregnancy and that also may be at some risk of exposure to a Sexually Transmitted Infection (STI).

Voluntary family planning services support people of reproductive age to make informed decisions and exercise personal choices to achieve their fertility goals. Healthy timing and spacing of pregnancies helps women have safer pregnancies and bear healthier children, thereby reducing child and maternal mortality in accordance with Millennium Development Goals (MDGs) 4 and 5. Family planning services also contribute to MDG 6 (combating HIV/AIDS, malaria, and other diseases) by educating clients about the use of primary prevention methods, such as condoms, for prevention of HIV and other sexually transmitted infections. (2)

\section{Background}

There are approximately 55 thousand people living with HIV in Nepal.And significant proportion $(86 \%)$ of this infection lies in adult men and women of reproductive age whose need for FP services is still unmet. The unmet need for contraception is not just the issue of people living with HIV, but also other most at risk population (MARPs)-female sex workers, people who inject drugs (PWID), migrants. The persistent "unmet need" for FP in general, with the increasing demand for FP by people living with HIV/AIDs and other high risk populationrequires that FP information and services be provided within HIV services-from voluntary counseling and testing to care and treatment.

FP and HIV/AIDS control are the two successful and priority one programs in Nepal. FP services are delivered through various government and nongovernmental service delivery outlets comprising hospital, NGOs, clinics and pharmacies.
HIV/AIDs related services are delivered through governmental as well as non governmental organization which offers services to risk population at risk with their different approach of service delivery. The utilization of FP services by this high risk population is low because of low access to existing FP service delivery outlets, due to fear of disclosure of their HIV status or activity, lack of friendly environment and long waiting hours. The best approach that would address all these problems came with idea of FP, HIV service integration in Nepal.

Integration of FP services with HIV related services in Nepal was pioneered in United States Agency for International Development (USAID) (3) funded Saath-Saath Project (SSP) in Nepal (4) though internationally it was practiced since a long time. SSP delivers quality services through expanded integrated health services (EIHS) centers to most-at-risk populations. EIHS centers are designed as such that all HIVrelated services are provided under one roof using integrated approach. Female sex workers (FSW), clients of FSW, People living with HIV (PLHIV), Migrants and spouse of migrants are the population for whom the integrated FP,HIV services are provided by SSP. (5) Findings of recent Integrated Bio Behavioral Surveys (IBBS) done among the FSWs in 2011 in Kathmandu, Pokhara and 22 Terai districts along the major highway showed that the condom use by FSWs with regular non-paying partners like a boyfriend or husband is low. (6) At the same time use of the other contraceptives is also low. It indicates that FSWs are at additional risk of unwanted pregnancy; this increase the possibility of mother-to-child transmission of HIV among the HIV positive FSWs-this sencario undoubtedly speaks for the integration of FP-HIV sevices in Nepal.(7)

\section{Discussion}

In an era when approximately 40 million adults and children are living with HIV/AIDS and women of childbearing age account for nearly half of the infected population (8), family planning has a critical role to play in curbing the HIV/AIDS epidemic. For both HIV-positive and HIV-negative individuals, contraceptive barrier methods such as the male and female condom provide dual protection against unintended pregnancy and HIV transmission. Among HIV-infected

CORRESPONDENCE: Shiva Raj Mishra,Journal Office, Health Prospect Volume 11,Program Officer,Naulo Ghumti Nepal,EIHS-Tanahun,Saath-Saath Project, Kathmandu, Nepal. Email: shivarajmishra@gmail.com 
women, the prevention of unintended pregnancies is essential for preventing mother-to-child transmission of HIV and reducing the number of children orphaned when parents die of AIDS-related illnesses. (9)

If women do not know she has HIV, if she is not tested before her pregnancy she can transmit HIV to her son/daughter which otherwise she would not have got pregnant or opted methods to prevent mother to child transmission of HIV. Similar scenes play out every day around the world. In sub-Saharan Africa and Asia where the burden of HIV, unintended pregnancies, and maternal and infant mortality are highest (10), missed opportunities to meet health care needs, can be deadly.

Integrated family planning and HIV/AIDS services have the potential to create synergistic relationships between programs, reduce missed opportunities, and ultimately maximize the effectiveness and impact of services by providing comprehensive reproductive health care that holistically addresses clients' dual risks of HIV infection and unintended pregnancy. (4) FP services in Nepal are very effective and established program, provided through government and non-government outlets. (11) By developing a strong coordination mechanism with the utilization of the existing HIV service providers to provide FP services and vice versa-effective integrated FP HIV services can be provided to the population at need. (7)

FP services and HIV services have similar characteristics, target populations, and desired outcomes. Clients seeking HIV services and those seeking FP services share common needs and concerns, and integrating services will enable health care service providers to efficiently and comprehensively address them. HIV services are good entry points for most of the sexually transmitted infection and vice versa. Strong linkages will help to ensure that the FP needs and aspirations of all people living with HIV, most at risk populations are met. (3)

Very often, integrated services create an image of a facility where a client could have all of his/her health needs met during the first encounter; this may not be feasible however, depending on the service capacity FP-integrated HIV services may be offered at the same facility or location during the same operating hours by the same provider with additional training in FP service delivery with an effective referral system in place. (12)

Obviously, challenges are there in incorporating FP into existing HIV services. It adds time to counseling, can increase workload, and can increase client waiting time, particularly when staffing levels are low, may lead to low quality of delivered services, increased burden of work in staff. Record keeping, activity reporting, logistic delivery system can be overstretched-creates need for regular guidance and supervision on added FP component. It may overload the client with information than they are not able to absorb.

Advancing FP/HIV integration needs political commitment by demonstrating the health, social, and economic benefits that integrated services can offer. However, political support alone is not sufficient; programmatic guidance is also needed to improve the services. There needs to have have participation of people living with HIV, AIDS and youth in decision making who provide guidance in developing national plan and strategies for implementing integrated services. (13) Scientific evidences needs to collected priodically to scale up as well as support integration FP-HIV services.

\section{Conclusion}

Increasing HIV prevalence in Nepal is pressing hard the HIV/ AIDS service delivery system. The FP need of PLHIV, FSWs, PWID, Migrants who have limited access to FP outlets of government and other organizations are unmet which makes integration of FP into existing HIV service delivery system necessary. Effective coordination mechanisms, policy documents and guidelines, periodic researches to identify gaps are needed to foster effective link between FP and HIV services. Multi-sectoral coordination and participation by most at risk population in service design and delivery are ubiquitously important in the success of FP, HIV integration in Nepal. FP and HIV/AIDS control program are two successful national programs. Integration between these are expected to bring more fruits as integration will improve the access to FP services, efficiently and comprehensively address unintended pregnancy and HIV transmission among these key affected populations.

\section{References}

1. Frontiers PC. Legacy series:Integration of services New York: Population Council Frontiers

2. Foundation EGPA. Family Planning and HIV Integration: An Essential Strategy for Preventing Pediatric HIV and Protecting Maternal and Child Health Wasington,D.C.2011.

3. USAID. AIDSTAR:One case study series New York: USAID2011.

4. FHI360. Integration of Family Planning and HIV/AIDS Services. Durham: FHI 360; 2012; Available from: http://www.fhi360.org/ en/Topics/FPHIV.htm.

5. FHI360. Prevention Guideline for outreach and prevention Kathmandu: FHI 3602009.

6. New ERA/SACTS FN. Integrated Bio- behavioral surveillance among female sex workers in Pokhara, Kathmandu Valleys. Kathmandu: Family Health International 2011

7. FHI360. Standarad Operating Procedure for Family Planning Services Kathmandu: FHI 3602012.

8. UNAIDS. Global AIDS Response Progress Reporting 2012. Geneva 2011

9. Department of Health Services M. National Guidelines: Prevention of Mother to Child Transmission of HIV in Nepal DoHS, MoHP2008.

10. Unicef. Progress for Children New York: Unicef2008.

11. MoHP DoHS. Annual Report Kathmandu Department of Health Services 2010

12. Farrell BL. Family Planning-Integrated HIV Services: A Framework for Integrating Family Planning and Antiretroviral Therapy Services New York: USAID Acquire Project 2007.

13. Kenya. Integrating Family Planning into HIV Voluntary Counseling and Testing Services in Kenya:Progress to Date and Lessons Learned. 